Pacific Journal of Mathematic 


\section{ON STOPPING RULES AND THE EXPECTED SUPREMUM OF $S_{n} / T_{n}$}

\section{Michael J. Klass and Lawrence E. Myers}

Let $S_{n}$ and $T_{n}$ be $n$th partial sums of two independent sequences of i.i.d. random variables. $S_{1}$ and $T_{1}$ may have different distributions. Assume $0 \leqq E S_{1}<\infty, E T_{1}<\infty$ and $P\left[T_{1}>0\right]=1$. Let $\mathscr{B}_{n}$ be the $\sigma$-field generated by $S_{1}, T_{1}, \cdots, S_{n}, T_{n}$, and let $R_{\infty}$ be the collection of extended-valued stopping rules with respect to $\mathscr{B}_{1}, \mathscr{B}_{2}, \cdots$. It is shown that $E \sup _{n \geqq 1} S_{n} / T_{n}<\infty$ iff $\sup _{\tau \in R_{\infty}} E S_{\tau} / T_{\tau}<\infty$ iff $E S_{1} \log ^{+} S_{1}<\infty$ and $E\left(T_{1}^{-1}\right)<\infty$. The (random) cutoff points characterizing the optimal rules are easily obtained as fixed points of certain contraction mappings. A Markov walk generalization of the Chow and Robbins binomial stopping problem is viewed within the $S_{n} / T_{n}$ framework.

1. Introduction. Let $U, U_{1}, U_{2}, \cdots$ and $V, V_{1}, V_{2}, \cdots$ be independent random variables defined on a common probability space $(\Omega, \mathscr{F}, P)$. Assume the $U$ 's are nondegenerate and identically distributed with $0 \leqq E U<\infty$. Assume the $V$ 's are identically distributed with $P[V>0]=1$ and $E V<\infty$. Let $S_{n}=U_{1}+\cdots+U_{n}$ and $T_{n}=$ $V_{1}+\cdots+V_{n}$. Define the $\sigma$-fields $\mathscr{B}_{n}=\mathscr{B}\left(U_{1}, V_{1}, \cdots, U_{n}, V_{n}\right), \mathscr{B}_{n}^{\prime}=$ $\mathscr{B}\left(U_{1}, \cdots, U_{n}\right), \mathscr{B}_{n}^{\prime \prime}=\mathscr{B}\left(V_{1}, \cdots, V_{n}\right)$, and let $R_{\infty}, R_{\infty}^{\prime}, R_{\infty}^{\prime \prime}$ be the collections of extended-valued stopping rules (Definition 1 [8]) with respect to $\left\{\mathscr{B}_{n}\right\}_{n=1}^{\infty},\left\{\mathscr{B}_{n}^{\prime}\right\}_{n=1}^{\infty},\left\{\mathscr{B}_{n}^{\prime \prime}\right\}_{n=1}^{\infty}$, respectively. That is, $\tau \in R_{\infty}\left(R_{\infty}^{\prime}, R_{\infty}^{\prime \prime}\right)$ if and only if $[\tau=n] \in \mathscr{B}_{n}\left(\mathscr{B}_{n}^{\prime}, \mathscr{B}_{n}^{\prime \prime}\right)$ for all $n \geqq 1$ and $P[\tau=\infty]+\sum_{n=1}^{\infty} P[\tau=n]=$ 1. In order that our expected rewards be well defined, we follow the strong law and set $S_{\infty} / \infty, \infty / T_{\infty}, S_{\infty} / T_{\infty}$ equal to $E U, 1 / E V, E U / E V$, respectively. Unless otherwise mentioned, all suprema and infima are over $\{n: n \geqq 1\}$. We write $E \sup S_{n} / T_{n}$ for $E\left[\sup _{n \geqq 1}\left(S_{n} / T_{n}\right)\right]$.

It is well known (Burkholder [1] and McCabe and Shepp [9]) that

$$
E \sup S_{n} / n<\infty \Leftrightarrow E U \log ^{+} U<\infty \Leftrightarrow \sup _{\tau \in R_{\infty}^{\prime}} E S_{\tau} / \tau<\infty,
$$

and in this case an optimal stopping rule exists (Siegmund [10]), i.e., the last supremum in (1.1) is attained by some $\tau \in R_{\infty}^{\prime}$.

Operating under successively weaker conditions, Chow and Robbins [2], Teicher and Wolfowitz [11], Dvoretzky [6], Thompson, Basu and Owen [12], Davis [4], and Klass [8] have proved that the (unique) 
minimal optimal rule is to stop at the first time $n$ such that $S_{n} \geqq a_{n}$, where $\left\{a_{n}\right\}_{n=1}^{\infty}$ is the strictly increasing sequence of positive constants satisfying $a_{n} / n=\sup _{\tau \in R_{\dot{\delta}}} E\left[\left(a_{n}+S_{\tau}\right) /(n+\tau)\right]$.

One purpose of this paper is to generalize the above results to the reward sequence $S_{n} / T_{n}$. The independence suggests treating $S_{n}$ and $T_{n}$ separately, via the elementary inequality

$$
\left(E \inf n / T_{n}\right)\left(E \sup S_{n} / n\right) \leqq E \sup S_{n} / T_{n}
$$

$$
\leqq\left(E \sup n / T_{n}\right)\left(E \sup S_{n} / n\right)
$$

In light of (1.1) our attentions focus on $n / T_{n}$. In $\$ 2$ is proved a general result (Theorem 1) which implies that $E \sup n / T_{n}<\infty$ just in case $E\left(V^{-1}\right)<\infty$. Section 3 shows that $E \sup S_{n} / T_{n}<\infty$ iff $\sup _{\tau \in R_{\infty}} E S_{\tau} / T_{\tau}<$ $\infty$ iff $E U \log ^{+} U<\infty$ and $E\left(V^{-1}\right)<\infty$.

For future reference and some immediate methodology we recall here that

$\left\{S_{n} / n\right\}_{n=\infty}^{1}$ is a reversed martingale,

so that the conditional Jensen's inequality and independence imply

(1.4) $\quad\left\{n / T_{n}\right\}_{n=\infty}^{1}$ and $\left\{S_{n} / T_{n}\right\}_{n=\infty}^{1}$ are reversed submartingales.

Application of a well known submartingale inequality (Doob [5], p. 317) to (1.3) yields the sufficiency of $E U \log ^{+} U<\infty$ in (1.1). A possible approach to characterizing $E \sup S_{n} / T_{n}<\infty$ (or $E \sup n / T_{n}<\infty$ ) might then be to apply the same inequality to obtain the sufficient condition $E(U / V) \log ^{+}(U / V)<\infty\left(E V^{-1} \log ^{+}\left(V^{-1}\right)<\infty\right)$. As our results show, these conditions are not "sufficiently" weak. After all, $E V^{-1} \log ^{+}\left(V^{-1}\right)<$ $\infty$ precisely when $E \sup n^{-1} \sum_{i=1}^{n} V_{1}^{-1}<\infty$, and $n^{-1} \sum_{t=1}^{n} V_{i}^{-1}$ almost surely dominates $n / T_{n}$, by the inequality of the arithmetic and harmonic means. The underlying idea in the proof of Theorem 1 is the classical inequality relating the arithmetic and geometric means.

In $\$ 4$ we employ contractions to obtain the cutoff points which characterize the optimal rules. The situation is somewhat novel in that the optimal stopping times depend on the intrinsic times $k$ only through the values of $T_{k}$ at those times, and the cutoff points are themselves random, owing to dependence on the $T_{k}$. This section relies heavily on $\S \S 1$ and 2 of Klass [8].

In $\$ 5$ we indicate how a Markov chain generalization of the Chow and Robbins [2] example may be viewed as an $S_{n} / T_{n}$ problem. 
2. Expected suprema of inverse generalized means. For simplicity we now assume (w.l.o.g.) that $V_{k}(\omega)>0$ for all $k \geqq 1$ and all $\omega \in \Omega$. Let

$$
\begin{aligned}
& M_{n}(t, \omega)=\left(n^{-1} \sum_{k=1}^{n}\left(V_{k}(\omega)\right)^{t}\right)^{1 / t} \text { for } t \neq 0 \\
& M_{n}(0, \omega)=\lim _{t \rightarrow 0} M_{n}(t, \omega)=\left(\prod_{k=1}^{n} V_{k}(\omega)\right)^{1 / n} .
\end{aligned}
$$

For $n$ and $\omega$ fixed, $M_{n}(t, \omega)$ is an increasing function of $t$ (Chapter 2 of Hardy, Littlewood and Polya [7]).

For $r>0$ let $\|X\|_{r}=[E(|X| r)]^{1 / r}$ if the expectation is finite; other-. wise let $\|X\|_{r}=\infty$.

THEOREM 1. For all $t \geqq 0$ and $N \geqq 1$

$$
E\left(\sup _{n \geq N}\left[M_{n}(t, \omega)\right]^{-1}\right) \leqq\left\|V^{-1}\right\|_{1 / N}\left(2^{N}+N \log 2+1\right) .
$$

Consequently, for all $t \geqq 0$

$$
E\left(V^{-1}\right) \leqq E\left(\sup _{n \geqq 1}\left[M_{n}(t, \omega)\right]^{-1}\right) \leqq(3+\log 2) E\left(V^{-1}\right),
$$

whence $E\left(\sup _{n \geqq 1}\left[M_{n}(t, \omega)\right]^{-1}\right)<\infty$ for (all) $t \geqq 0$ if and only if $E\left(V^{-1}\right)<$ $\infty$. More generally,

$$
E\left(\sup _{n \geqq N}\left[M_{n}(t, \omega)\right]^{-1}\right)<\infty \text { for (all) } t>0
$$

$$
\text { if and only if } E \min _{1 \leqq J \leqslant N}\left(V_{J}^{-1}\right)<\infty \text {, }
$$

whereas

$$
E\left(\sup _{n \geqq N}\left[M_{n}(0, \omega)\right]^{-1}\right)<\infty \text { if and only if } E\left(V^{-1 / N}\right)<\infty
$$

Proof. First we establish (2.1). Since for $n$ and $\omega$ fixed and $t \geqq 0$ the $\left[M_{n}(t, \omega)\right]^{-1}$ are all majorized by the inverse geometric mean $\left[M_{n}(0, \omega)\right]^{-1}=\left(\prod_{k=1}^{n} V_{k}^{-1}\right)^{1 / n}$, it suffices to prove (2.1) for $t=0$.

Fix $N \geqq 1$. We may assume $\left\|V^{-1}\right\|_{1 / N}<\infty$. Let $C=E\left(V^{-1 / N}\right)$ and $B=\left[2 E\left(V^{-1 / N}\right)\right]^{N}$. Then 


$$
\begin{aligned}
E \sup _{n \geqq N}\left(\prod_{l=1}^{n} V_{l}^{-1 / n}\right) & =\int_{0}^{\infty} P\left[\sup _{n \geq N} \prod_{i=1}^{n} V_{t}^{-1 / n} \geqq y\right] d y \\
& \leqq B+\left[E\left(V^{-1 / N}\right)\right]^{N}+\sum_{n=N+1}^{\infty} \int_{B}^{\infty} P\left[\prod_{l=1}^{n} V_{t}^{-1 / N} \geqq y^{n / N}\right] d y \\
& \leqq B\left(1+2^{-N}\right)+\sum_{n=N+1}^{\infty} C^{n} \int_{B}^{\infty} y^{-n / N} d y \\
& =\left\|V^{-1}\right\|_{1 / N}\left(2^{N}+N \log 2+1\right) .
\end{aligned}
$$

This proves (2.1), from which (2.2) and (2.4) follow readily. To prove (2.3) note that for $t>0$

$$
N^{-1 / t} \max _{1 \leq l \leq N} V_{J} \leqq M_{N}(t, \omega) \leqq \max _{1 \leqq J \leqq N} V_{J}
$$

Hence for $t>0$

$$
\begin{aligned}
E \min _{1 \leqq J \leqq N}\left(V_{1}^{-1}\right) & \leqq E\left(\sup _{n \geq N}\left[M_{n}(t, \omega)\right]^{-1}\right) \\
& \leqq N^{1 / t} E \min _{1 \leqq I \leqq N}\left(V_{1}^{-1}\right)+E\left(\sup _{n>N}\left[M_{n}(t, \omega)\right]^{-1}\right) .
\end{aligned}
$$

We may assume $E \min _{1 \leqq \jmath \leqq N}\left(V_{1}^{-1}\right)<\infty$, in which case $\lim _{y \rightarrow \infty} y\left(P\left(V^{-1}>y\right)\right)^{N}=\lim _{y \rightarrow \infty} y P\left[\min _{1 \leq_{j} \leq N} V_{j}^{-1}>y\right]=0$. We may conclude that $E\left(V^{-1 / \alpha}\right)<\infty$ for any $\alpha>N$. Take $\alpha=N+1$ and use (2.1) to complete the proof.

Taking $t=1$ in (2.2) yields

Corollary 1. $E \sup n / T_{n}<\infty \Leftrightarrow E\left(V^{-1}\right)<\infty$.

REMARK 1. To illustrate the (qualitative) sharpness of (2.1) for $t=1$, fix $N \geqq 2$ and let $V$ be a gamma random variable with mean and variance both equal to $1 /(N-1)$. Then $E n / T_{n}=\infty$ for $1 \leqq n<N$, while by (2.1) $E \sup _{n \geqq N} n / T_{n}<\infty$.

To underline the distinction between (2.3) and (2.4), take $N \geqq 2$ and $P\left[V^{-1}>y\right]=\left(y^{1 / N} \log (e y)\right)^{-1}$ for $y \geqq 1$. Then

$$
E\left(V^{-1 / N}\right)=1+\int_{1}^{\infty}\left(y N \log \left(e^{1 / N} y\right)\right)^{-1} d y=\infty,
$$

while

$$
E \min _{1 \leqq j \leq N}\left(V_{l}^{-1}\right)=1+\int_{1}^{\infty}\left(P\left(V^{-1}>y\right)\right)^{N} d y<\infty .
$$


Whenever $E\left(V^{-1}\right)=\infty>E\left(V^{-1 / N}\right)$, Theorem 1 yields that $E \sup _{n \geqq 1} n / T_{n}=\infty>E \sup _{n \geqq N} n / T_{n}$, so that the infinite expected supremum owes exclusively to the behavior of the first few terms. Our next result sheds additional light on this.

THEOREM 2. Let $V, V_{1}, V_{2}, \cdots$ be i.i.d. nonnegative random variables with $P[V>0]>0$. Then

$$
E \sup n /\left(b+T_{n}\right)<\infty \text { for each } b>0 .
$$

Proof. We use ladder variables to transform the given reward sequence to an $S_{n} / n$ reward sequence.

There exists $c>0$ such that $P[V \geqq c] \geqq c$. Let $\tau(0)=0$. Having defined $\tau(0), \cdots, \tau(k)$, let $\tau(k+1)=1$ st $n \quad$ s.t. $\quad V_{1}+\cdots+V_{n} \geqq$ $c+V_{1}+\cdots+V_{\tau(k)}$. Then $T_{\tau(k)} \geqq k c$. The random variables $\tau(k)$ (for $k \geqq 1$ ) are sums of $k$ i.i.d. ladder variables $q_{1}, \cdots, q_{k}$. Note that $P\left[q_{1}>n\right]=P[\tau(1)>n] \leqq P\left[\bigcap_{1=1}^{n}\left\{V_{1}<c\right\}\right]=[P(V<c)]^{n} \leqq(1-c)^{n}$, so that all moments of $q_{1}$ are finite. Further,

$$
\begin{aligned}
E \sup n /\left(b+T_{n}\right) & =E \sup _{k \geqq 0} \sup _{\tau(k)<n \geqq \tau(k+1)} n /\left(b+T_{n}\right) \\
& \leqq E \sup _{k \geqq 0} \tau(k+1) /(b+k c) \\
& \leqq(1 / b) E \tau(1)+(2 / c) E \sup _{k \geqq 1} \tau(k) / k,
\end{aligned}
$$

which is finite by (1.1).

REMARK 2. We conclude this section by mentioning another condition equivalent to $E \sup n / T_{n}<\infty$. One can show that

$$
[r /(r+1)] E Y_{1} \sup Y_{n}^{-(r+1)} \leqq E \sup Y_{n}^{-r} \leqq E Y_{1} \sup Y_{n}^{-(r+1)}
$$

for any $r>0$ and any positive reversed martingale $\cdots Y_{2}, Y_{1}$ (the upper bound is trivial; the lower bound follows from an integration by parts, inequality (3.4") of Doob [5, p. 314], and Fubini's theorem). It follows from (2.6) and (1.3) that

$$
\sup n / T_{n} \in L_{1}(P) \quad \text { iff } \quad T_{1}^{1 / 2} \sup n / T_{n} \in L_{2}(P) \text {. }
$$

3. $E \sup S_{n} / T_{n}<\infty \Leftrightarrow E U \log ^{+} U<\infty$ and $E\left(V^{-1}\right)<$ $\infty$. The following lemma is a consequence of the strong law. The corollary follows from the lemma and (1.2). 
LEMMA 1. $P\left[\inf n / T_{n}=0\right]=0$ and $0<E \inf n / T_{n}<\infty$.

Corollary 2. $E \sup S_{n} / T_{n}=\infty$ whenever $E \sup S_{n} / n=\infty$.

THEOREM 3. The following are equivalent.

(i) $\sup _{\tau \in R_{\infty}} E S_{\tau} / T_{\tau}<\infty$

(ii) $E \sup S_{n} / T_{n}<\infty$

(iii) $E \sup S_{n} / n<\infty$ and $E \sup n / T_{n}<\infty$

(iv) $E U \log ^{+} U<\infty$ and $E\left(V^{-1}\right)<\infty$.

Proof. (iii) and (iv) are equivalent by (1.1) and Corollary 1. (iii) implies (ii) by (1.2). (ii) implies (i) since $E \sup Y_{n} \geqq \sup _{\tau \in R_{\infty}} E Y_{\tau}$ for any reward sequence $\left\{Y_{n}\right\}_{n=1}^{\infty}$. The chain will be completed by showing the inverse of $[(i v) \Rightarrow(i)]$.

Suppose first that $E\left(V^{-1}\right)=\infty$. Define $\tau \in R_{\infty}^{\prime}$ by $\tau=1$ if $U_{1}>0, \tau=\infty$ otherwise. Then $E S_{\tau} / T_{\tau}=\infty$ since $P\left[U_{1}>0\right]>0$.

Now suppose $E U \log ^{+} U=\infty$. Then $\sup _{t \in R_{\infty}} E S_{t} / t=\infty$ [9]. It follows that for every $m \geqq 1$ there exists $\tau_{m} \in R_{\infty}^{\prime}$ such that $E S_{\tau_{m}} / \tau_{m}>$ $m / E \inf \left(n / T_{n}\right)$; Lemma 1 has been invoked here $\left(0<E\right.$ inf $\left.n / T_{n}<\infty\right)$. Because each $\tau_{m}$ is independent of the $V_{\imath}$, and $\mathscr{B}\left(S_{1}, T_{1}, \cdots, S_{m}, T_{m}\right) \supseteq$ $\mathscr{B}\left(S_{1}, \cdots, S_{m}\right)$ for every $m$, we have

$$
\begin{aligned}
\sup _{\tau \in R_{\infty}} E S_{\tau} / T_{\tau} & \geqq \sup _{\tau \in R_{\infty}^{\infty}} E S_{\tau} / T_{\tau} \geqq \sup _{m \geqq 1} E\left[\left(S_{\tau_{m}} / \tau_{m}\right) \inf _{n \geqq 1} n / T_{n}\right] \\
& =\sup _{m \geqq 1}\left[E\left(S_{\tau_{m}} / \tau_{m}\right) E \inf _{n \geqq 1} n / T_{n}\right]=\sup _{m \geqq 1} m=\infty .
\end{aligned}
$$

This completes the proof.

4. The form of the optimal rule. We assume throughout this section that $E\left(V^{-1}\right)$ and $E U \log ^{+} U$ are both finite. Our return sequences $Y_{n}(a, b)$ are defined by $Y_{n}(a, b)=\left(a+S_{n}\right) /\left(b+T_{n}\right)$, a real, $b \geqq 0$. Since $Y_{n}(a, b) \stackrel{\text { a.s. }}{\longrightarrow} E U / E V$ and $T_{n} \uparrow \infty$ a.s., we set $Y_{\infty}(a, b)=$ $E U / E V$ and $T_{\infty}=\infty$. By the results of $\S 3, E \sup Y_{n}(a, b)<\infty$. We thus see that assumptions $A_{1}, A_{2}, A_{3}$ of Klass [8] hold for our $Y_{n}(a, b)$, so that the entirety of $\$ 1$ there is applicable. In particular

$$
M_{b}(a)=\sup _{\tau \in R_{\infty}} E\left(a+S_{\tau}\right) /\left(b+T_{\tau}\right)
$$

is well-defined, finite, and attained by some $\tau \in R_{\infty}$ (Klass [8], Theorem 1).

We omit the proof of the following lemma ( $E V<\infty$ is used). 
LEMMA 2. For each $b \geqq 0$ there exists $\epsilon(b)>0$ such that for any $\tau \in R_{\infty}$

$$
0 \leqq E\left[1 /\left(b+T_{\tau}\right)\right] \leqq E\left[1 /\left(b+T_{1}\right)\right]=1 /(b+\epsilon(b))
$$

If $P[\tau<\infty]>0$ the leftmost inequality is strict.

REMARK 3. In the $S_{\tau} / \tau$ problem $\left(\tau \in R_{\infty}^{\prime}\right)$, the form of the minimal strictly semi-optimal rule (Definitions 4 and 5 of Klass [8]) is dictated by the fact that for each $n \geqq 0$ there is a unique $a_{n}$ such that $M_{n}\left(a_{n}\right)=$ $a_{n} / n$. Our result, in addition to being more general, is obtained with a considerable economy of effort over earlier ones through the observation that the maps $a \rightarrow b M_{b}(a)$ contract the reals.

THEOREM 4. Fix $b \geqq 0 . \quad M_{b}(a)>E U / E V \geqq 0$ for each $a . \quad M_{b}(a)$ is a continuous strictly increasing function of $a . \quad b M_{b}$ is a contraction of the reals, and so has a unique fixed point $a_{b}\left(b M_{b}\left(a_{b}\right)=a_{b}\right)$.

Proof. The theorem is proved with the appropriate modifications of the proof of Lemma 8, page 729 of Klass [8]. Fix $b \geqq 0$.

For the first assertion, it suffices to show that $P\left[\sup \left(a+S_{n}\right) /(b+\right.$ $\left.\left.T_{n}\right)>E U / E V\right]=1$ for any $a$. But $\left(a+S_{n}\right) /\left(b+T_{n}\right)>E U / E V$ if and only if $\sum_{i=1}^{n}\left(U_{l}-(E U / E V) V_{t}\right)>b(E U / E V)-a$. Since a nondegenerate mean zero random walk almost surely exceeds any real number infinitely often, the first assertion is proved.

Again fix $b \geqq 0$, let $a_{1}<a_{2}$, and let $\tau_{1}$ attain $M_{b}\left(a_{1}\right), i=1,2$. Then $P\left[\tau_{\imath}<\infty\right]>0$ since $M_{b}\left(a_{\imath}\right)>E U / E V, i=1,2$, and two applications of Lemma 2 yield

$$
\begin{aligned}
0 & <\left(a_{2}-a_{1}\right) E\left[1 /\left(b+T_{\tau_{1}}\right)\right] \leqq M_{b}\left(a_{2}\right)-M_{b}\left(a_{1}\right) \\
& \leqq\left(a_{2}-a_{1}\right) E\left[1 /\left(b+T_{\tau_{2}}\right)\right] \leqq\left(a_{2}-a_{1}\right) /[b+\epsilon(b)] .
\end{aligned}
$$

The continuity of $M_{b}$ follows, as does the last assertion of the theorem:

$$
\left|b M_{b}\left(a_{2}\right)-b M_{b}\left(a_{1}\right)\right| \leqq \frac{b}{b+\epsilon(b)}\left|a_{2}-a_{1}\right|
$$

Lemmas 6 and 7 and Remark 2 of Klass [8] carry over in straightforward fashion to our case, culminating in 
LEMMA 3. For $b \geqq 0$ :

(i) $a<a_{b} \Rightarrow b M_{b}(a)>a$

(ii) $a>a_{b} \Rightarrow b M_{b}(a)<a$

(iii) $\epsilon>0 \Rightarrow a_{b+\epsilon}>a_{b}$.

Rather than introduce randomization (which is "unnecessary"; see Theorem 5.3, p. 111 of Chow, Robbins and Siegmund [3]) and determine up to equivalence the collection of all $\tau$ which attain $M_{b}(a)$, we content ourselves with exhibiting one such $\tau$. The situation is somewhat novel in that the optimal stopping time depends on intrinsic time $k$ only through the values of the $T_{k}$ at those times, and the cutoff points $a_{T_{k}}$ are themselves random. Then $a_{b}$ in Theorem 5 are in accordance with those of Theorem 4.

THEOREM 5. Given a real, $b \geqq 0$, define $\tau \in R_{\infty}$ by

$$
\begin{aligned}
\tau & =\min \left\{k: a+S_{k}>a_{b+T_{k}}\right\} \\
& =\infty \quad \text { if } a+S_{k} \leqq a_{b+T_{k}} \text { for all } k .
\end{aligned}
$$

Then $E\left(a+S_{\tau}\right) /\left(b+T_{\tau}\right)=M_{b}(a)$.

Proof. Clearly $\tau \in R_{\infty}$. To show that $\tau$ is optimal for the reward sequence $Y_{n}(a, b)$, it suffices to show that $\tau$ is minimal strictly semioptimal (Definitions 4 and 5 and Theorem 6 of Klass [8]).

Suppose $S_{n}=s_{n}, T_{n}=t_{n}$ and $\tau$ instructs us to stop at time $n$ for the reward $\left(a+s_{n}\right) /\left(b+t_{n}\right)>a_{b+t_{n}} /\left(b+t_{n}\right)$. By continuing we would expect to get at most $M_{b+t_{n}}\left(a+s_{n}\right)$, which is strictly less than $\left(a+s_{n}\right) /\left(b+t_{n}\right)$, by (ii) of Lemma 3. Hence $\tau$ is strictly semi-optimal.

The proof that $\tau$ is minimal (strictly semi-optimal) is as in the proof of Theorem 7 of Klass [8, p. 734], with $a_{n+k}$ replaced by $a_{b+T_{k}}$.

5. A Markov walk example. The following example generalizes the fair coin tossing problem treated in Chow and Robbins [2]. Let $\left\{X_{k}\right\}_{k=1}^{\infty}$ be a $\{0,1\}$-valued stationary Markov chain with $P\left[X_{k+1}=1 \mid X_{k}=0\right]=p=1-q$ and $P\left[X_{k+1}=0 \mid X_{k}=1\right]=p^{\prime}=1-q^{\prime}$. In order that the chain have stationary initial distribution we must have $P\left[X_{1}=1\right]=a=p /\left(p+p^{\prime}\right)$. We consider the optimal stopping problem with reward sequence $S_{n}^{*} / n=\left(X_{1}+\cdots+X_{n}\right) / n$. Let $v=\sup _{\tau \in R_{z}^{*}} E S_{\tau}^{*} / \tau$, where $R_{\infty}^{*}$ is the collection of stopping rules w.r.t. $\left\{\mathscr{B}\left(X_{1}, \cdots, X_{n}\right)\right\}_{n=1}^{\infty}$.

Clearly any optimal rule has $\tau=1$ if $X_{1}=1$ (otherwise $\tau$ is not regular; see Definition 2 and Theorem 2 of Klass [8]).

Now suppose $X_{1}=0$. We thrust independence into the picture as follows. Suppose the statistician gets to see the data, not a digit ( 0 or 1$)$ 
at a time, but in blocks (more formally, instead of observing the original $X_{i}$, he views the sojourn times $V_{1}, U_{1}, V_{2}, U_{2}, \cdots$, where $V_{1}\left(U_{i}\right)$ is the time spent in the $i$ th visit to $\{0\}(\{1\}))$. The idea here is that, in the context of the original "game", it is clearly more profitable to stop at the end of some string of 1's as opposed to stopping in the middle of a 1-block or somewhere in a 0 -block.

So let $U, U_{1}, U_{2}, \cdots$ be i.i.d. geometric r.v.'s with $P[U=k]=$ $\left(q^{\prime}\right)^{k-1} p^{\prime}, k \geqq 1$, and let $V, V_{1}, V_{2}, \cdots$ be i.i.d. geometric r.v.'s with $P[V=k]=q^{k-1} p$. Then the foregoing heuristics show that

$$
\begin{aligned}
v & \leqq a+(1-a) E \sup \left[S_{n} /\left(S_{n}+T_{n}\right)\right] \\
& \leqq a+(1-a)\left(E \sup S_{n} / T_{n}\right) /\left(E \sup S_{n} / T_{n}+1\right) .
\end{aligned}
$$

Here we have used Jensen's inequality and the fact that $f(x)=x /(x+1)$ is concave increasing for $x>0$. In this way an upper bound on $E \sup S_{n} / T_{n}$ may be employed in majorizing $v$.

For example, one may use Theorem 3.4, p. 317 of Doob [5], together with the fact that $\left\{S_{n} / T_{n}\right\}_{n=\infty}^{1}$ is a reversed submartingale, to obtain

$$
E \sup S_{n} / T_{n} \leqq[e /(e-1)]\left[1+E(U / V) \log ^{+}(U / V)\right]
$$

\section{REFERENCES}

1. D. L. Burkholder, Successive conditional expectation of an integrable function, Ann. Math. Statist., 33 (1962), 887-893.

2. Y. S. Chow and H. Robbins, On optimal stopping rules for $S_{n} / n$, Illinois J. Math., 9 (1965), 444-454.

3. Y.S. Chow, H. Robbins and D. Siegmund, Great Expectations: The Theory of Optimal Stopping, Houghton Mifflin, Boston, (1971).

4. B. Davis, Moments of random walks having infinite variance and the existence of certain optimal stopping rules for $S_{n} / n$, Illinois J. Math., 17 (1973), 75-81.

5. J. L. Doob, Stochastic Processes, Wiley, New York, (1953).

6. Aryeh Dvoretzky, Existence and properties of certain optimal stopping rules, Proc. Fifth Berkeley Symp. Math. Statist. Prob., 1 (1967), 441-452, University of California Press.

7. Hardy Littlewood and Polya, Inequalities, 2nd ed., Academic Press, (1951).

8. M. J. Klass, Properties of optimal extended-valued stopping rules for $S_{n} / n$, Ann. Prob., 1 (1973), 719-757.

9. B. J. McCabe and L. A. Shepp, On the supremum of $S_{n} / n$, Ann. Math. Statist., 41 (1970), 2166-2168.

10. D. O. Siegmund, Some problems in the theory of optimal stopping rules, Ann. Math. Statist., 38 (1967), 1627-1640.

11. H. Teicher and J. Wolfowitz, Existence of optimal stopping rules for linear and quadratic rewards,

Z. Warhscheinlichkeitstheorie und Verw. Gebiete., 5 (1966), 361-368. 
12. M. E. Thompson, A. K. Basu and W. L. Owen, On the existence of the optimal stopping rule in the $S_{n} / n$ problem where the second moment is infinite, Ann. Math. Statist., 42 (1971), 1936-1942.

Received April 20, 1976. The first author was partially supported by the Miller Foundation for Basic Research. The second author was partially supported by grant CA14236 from the National Cancer Institute.

Department of Statistics

UNIVERSITY OF CALIFORNIA

BERKELEY, CA 94720

AND

Deparment of Community and Family Medicine

DUKe University Medical Center

DURHARM, NC 27710 


\section{PACIFIC JOURNAL OF MATHEMATICS}

\section{EDITORS}

RICHARD ARENS (Managing Editor)

University of California

Los Angeles, CA 90024

R. A. BeAumont

University of Washington

Seattle, WA 98105

C. C. MOORE

University of California

Berkeley, CA 94720
J. DUGUNDJI

Department of Mathematics

University of Southern California

Los Angeles, CA 90007

R. FINN AND J. MILGRAM

Stanford University

Stanford, CA 94305

\section{ASSOCIATE EDITORS}
E. F. BECKENBACH
B. H. NEUMANN
F. WOLF
K. YoshidA

\section{SUPPORTING INSTITUTIONS}

UNIVERSITY OF BRITISH COLUMBIA

UNIVERSITY OF SOUTHERN CALIFORNIA

CALIFORNIA INSTITUTE OF TECHNOLOGY

STANFORD UNIVERSITY

UNIVERSITY OF CALIFORNIA

UNIVERSITY OF HAWAII

MONTANA STATE UNIVERSITY

UNIVERSITY OF TOKYO

UNIVERSITY OF NEVADA

UNIVERSITY OF UTAH

NEW MEXICO STATE UNIVERSITY

OREGON STATE UNIVERSITY

UNIVERSITY OF OREGON

OSAKA UNIVERSITY

WASHINGTON STATE UNIVERSITY

UNIVERSITY OF WASHINGTON

AMERICAN MATHEMATICAL SOCIETY

The Supporting Institutions listed above contribute to the cost of publication of this Journal, but they are not owners or publishers and have no responsibility for its contents or policies.

Mathematical papers intended for publication in the Pacific Journal of Mathematics should be in typed form or offset-reproduced (not dittoed), double spaced with large margins. Underline Greek letters in red, German in green, and script in blue. The first paragraph or two must be capable of being used separately as a synopsis of the entire paper. Items of the bibliography should not be cited there unless absolutely necessary, in which case they must be identified by author and Journal, rather than by item number. Manuscripts, in duplicate, may be sent to any one of the four editors. Please classify according to the scheme of Math. Reviews, Index to Vol. 39. All other communications should be addressed to the managing editor, or Elaine Barth, University of California, Los Angeles, California, 90024.

100 reprints are provided free for each article, only if page charges have been substantially paid. Additional copies may be obtained at cost in multiples of 50 .

The Pacific Journal of Mathematics is issued monthly as of January 1966. Regular subscription rate: $\$ 72.00$ a year (6 Vols., 12 issues). Special rate: $\$ 36.00$ a year to individual members of supporting institutions.

Subscriptions, orders for numbers issued in the last three calendar years, and changes of address should be sent to Pacific Journal of Mathematics, 103 Highland Boulevard, Berkeley, California, 94708.

PUBLISHED BY PACIFIC JOURNAL OF MATHEMATICS, A NON-PROFIT CORPORATION

Printed at Jerusalem Academic Press, POB 2390, Jerusalem, Israel. 


\section{Pacific Journal of Mathematics}

\section{Vol. 75, No. $2 \quad$ October, 1978}

Susan Jane Zimmerman Andima and W. J. Thron, Order-induced

topological properties ................................... 297

Gregory Wade Bell, Cohomology of degree 1 and 2 of the Suzuki groups . . 319

Richard Body and Roy Rene Douglas, Rational homotopy and unique

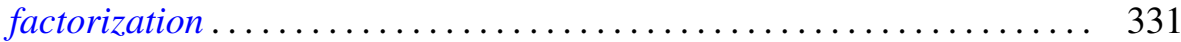

Frank Lewis Capobianco, Fixed sets of involutions ................. 339

L. Carlitz, Some theorems on generalized Dedekind-Rademacher sums .... 347

Mary Rodriguez Embry and Alan Leslie Lambert, The structure of a special class of weighted translation semigroups .....................

Steve Ferry, Strongly regular mappings with compact ANR fibers are

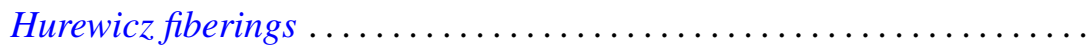

Ivan Filippenko and Marvin David Marcus, On the unitary invariance of the

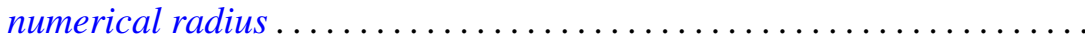

$\mathrm{H}$. Groemer, On the extension of additive functionals on classes of convex

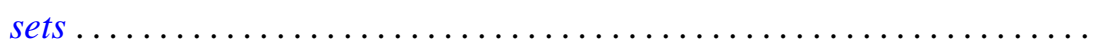

Rita Hall, On the cohomology of Kuga's fiber variety ............... 411

H. B. Hamilton, Congruences on $\mathrm{N}$-semigroups ................. 423

Manfred Herrmann and Rolf Schmidt, Regular sequences and lifting

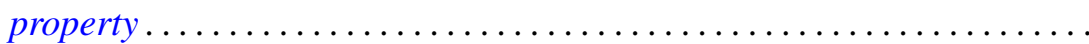

James Edgar Keesling, Decompositions of the Stone-Čech compactification which are shape equivalences .....................

Michael Jay Klass and Lawrence Edward Myers, On stopping rules and the expected supremum of $S_{n} / T_{n}$

Ronald Charles Linton, $\lambda$-large subgroups of $C_{\lambda}$-groups

William Owen Murray, IV and L. Bruce Treybig, Triangulations with the free cell property ............................

Louis Jackson Ratliff, Jr., Polynomial rings and $H_{i}$-local rings ...

Michael Rich, On alternate rings and their attached Jordan rings....

Gary Sampson and H. Tuy, Fourier transforms and their Lipschitz

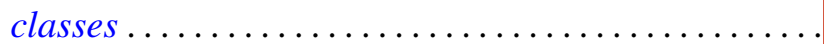

Helga Schirmer, Effluent and noneffluent fixed points on dendrites ...

Daniel Byron Shapiro, Intersections of the space of skew-symmetric maps

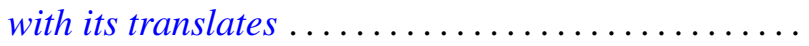

Edwin Spanier, Tautness for Alexander-Spanier cohomology ...

Alan Stein and Ivan Ernest Stux, A mean value theorem for binary digits ...

Franklin D. Tall, Normal subspaces of the density topology . .

William Yslas Vélez, Prime ideal decomposition in $F\left(\mu^{1 / p}\right) \ldots$ 